\title{
Fabrication of a porous alumina mask on the large surface area of a semi-insulating semiconductor substrate
}

\author{
Chieko TAKIZAWA, Seiichi KATO, ${ }^{* \dagger}$ Atsushi GoTO, Hideaki KITAZAWA, ${ }^{*}$ \\ Naoki IKEDA** and Yoshimasa SUGIMOTO ${ }^{* *}$ \\ Nano Characterization Unit, National Institute for Materials Science, 3-13 Sakura, Tsukuba, Ibaraki 305-0003, Japan \\ *Quantum Beam Unit, National Institute for Materials Science, 1-2-1 Sengen, Tsukuba, Ibaraki 305-0047, Japan \\ ${ }^{* *}$ Nanotechnology Innovation Station, National Institute for Materials Science, 1-2-1 Sengen, Tsukuba, Ibaraki 305-0047, Japan
}

\begin{abstract}
We describe a method for fabricating a large-scale homogeneous porous alumina membrane as a mask for dry etching of the large surface area (of the order of one square centimeter) of a semi-insulating semiconductor substrate. It employs direct anodization of the aluminum layer deposited on the substrate, which is simple, therefore, suitable for high-throughput productions. However, the process with a non-conductive semiconductor substrate suffers from the issues of quality control of the pores caused by nonuniform current flow in the aluminum layer in the anodization process. Hence, we have implemented a new fitting to the anode that provides uniform current flow into the aluminum layer, which allows us to fabricate highly homogeneous nanopores on the large-scale alumina membrane.
\end{abstract}

(C)2013 The Ceramic Society of Japan. All rights reserved.

Key-words : Anodization, Porous alumina, Semiconducting substrate, Dry etching, Current uniformization

[Received October 26, 2012; Accepted March 23, 2013]

\section{Introduction}

Fabrication of regularly ordered nanopores on the surface of a semiconductor is one of the fundamental processes for semiconductor devise fabrication and is widely used in the MEMS, ${ }^{1)}$ solar cells ${ }^{2)}$ and photonics ${ }^{3)}$ applications. One of the common schemes for this process is a dry etching with a mask prepared by photo-lithography. The photo-lithography is useful for small area of the order of square micrometers; however, it is not suitable or even unrealistic to apply for the large area of the order of square millimeters from the time and cost-performance point of view. Instead, one can use the electrochemical process called "anodization", where a porous alumina membrane is fabricated by anodizing an aluminum film in an acid solution. It provides a simple and low-cost alternative for the preparation of a largescale porous mask. ${ }^{4)}$

In the anodization process, there are two choices for setting a porous alumina membrane on the substrate: i.e., indirect ${ }^{5)}$ and direct processes. ${ }^{6}$ In the indirect process, the porous alumina membrane is prepared separately and set on the substrate afterwards. This process has been widely used because of the good quality of the porous membranes; however, it had required the toxic mercury (II) chloride solution to peel off the porous alumina from the aluminum film, which had caused low processibility. Recently, the method of creating free-standing alumina membranes has been developed for the indirect process, ${ }^{7}$ ) which provides a promising direction to circumvent the issue of toxic solutions for peeling.

The direct process, on the other hand, is characterized by the anodization of the aluminum film directly deposited on the semiconductor substrate. It is beneficial in that no process for setting a membrane on the substrate is required, which results in high processibility, thus, high throughput. The problem is that it is

\footnotetext{
Corresponding author: S. Kato; E-mail: KATO.Seiichi@nims.go.jp
}

difficult to control the quality of the membrane on a nonconductive substrate; the anodization process tends to proceed nonuniformly, which hinders the formation of uniform nanopores. We have found that the quality of the pores largely depends on the electric current flow in the aluminum layer; therefore, we have implemented a new fitting to the anode that provides uniform current flow in the aluminum layer, resulting in the improvement of the quality of the membrane. In this article, we report on the details of the fabrication procedure along with the new fitting.

\section{Fabrication method}

The procedure of the direct process is shown in Fig. 1. In our experiment, we used a single-crystal wafer of semi-insulating InP doped with $\mathrm{Fe}(350 \mu \mathrm{m}$ thick, Acrotec) as a semiconducting substrate. On this substrate, aluminum metal was deposited in a vacuum to form aluminum thin film a few micrometers thick, which was followed by the anodization of the whole sample in $0.3 \mathrm{M}$ oxalic acid solution for $2 \mathrm{~min}$ at $20^{\circ} \mathrm{C}$ with the applied voltage of $40 \mathrm{~V}$. In this process, we used a new fitting to the anode to secure the uniform current flow inside the anode, which will be described in the next section. Consequently, we obtained a completely oxidized alumina layer with numerous nanopores in it. Then, we soaked the sample in the mixture of $6 \mathrm{wt} \%$ phosphoric acid and $1.8 \mathrm{wt} \%$ chromic acid at $60^{\circ} \mathrm{C}$ for $1 \mathrm{~min}$. This process has the effects of pore widening and the removal of the barrier layers remaining at the bottoms of the pores, which is very effective to obtain well-ordered nanopores. ${ }^{8)}$ Figure 2(a) shows the scanning electron microscope (SEM) image of the sample at the end of this process. One finds that the nanopores completely penetrate the alumina layer and reach the InP substrate.

Subsequently, the sample was subjected to the inductive coupled plasma reactive ion etching (ICP-RIE) process for $5 \mathrm{~min}$ under the following conditions: gas flow rate: $\mathrm{Cl}_{2} / \mathrm{Ar}=1 / 1$ 
(1) Aluminum deposition

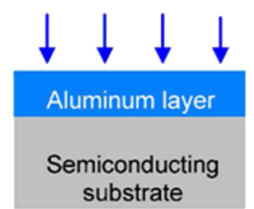

(2) Anodization in acids Porous alumina Pon

(3) Pore widening

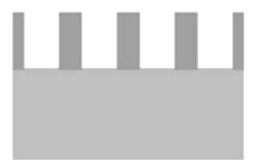

Fig. 1. Nanopore fabrication on the surface of a semiconducting substrate using a porous alumina mask (direct process).
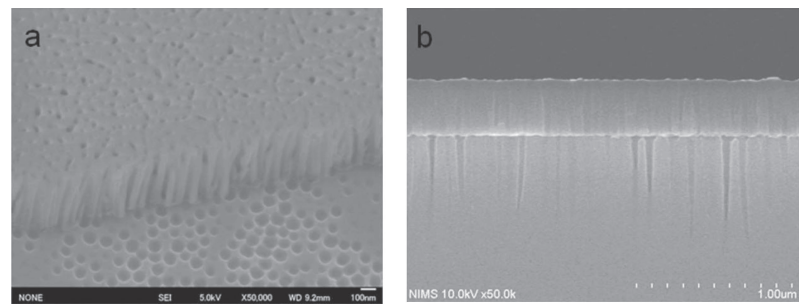

Fig. 2. (a) SEM image of the top part of the sample before the dry etching process. A part of the surface of the InP substrate is exposed for demonstration purpose (lower part of the image). (b) Cross-sectional SEM image after the dry etching process. The upper and the lower layers correspond to the porous alumina and the InP substrate, respectively.

sccm, pressure: $0.1 \mathrm{~Pa}$, substrate temperature: $200^{\circ} \mathrm{C}, \mathrm{ICP}$ : $300 \mathrm{~W}$, bias: $150 \mathrm{~W}$. The process results in the formation of regularly ordered nanopores on the surface of the InP substrate. Figure 2(b) shows the cross-sectional SEM image of the sample after this process. One finds the formation of nanopores in the InP substrate. After removing the residual alumina film on the surface with a PTFE tape coated with a silicon-based material, we finally obtained numerous nanopores of 50-60 $\mathrm{nm}$ in diameter and approximately $0.5 \mu \mathrm{m}$ in depth on the semiconducting substrate about $1 \mathrm{~cm}$ square.

The diameter and the depth of the pores are the important specifications determined by the requirements of the intended use. In principle, the diameter is controlled through the conditions in the anodization process. The depth, on the other hand, is determined substantially by the thickness of the alumina mask before the dry etching. This is because the etching can be performed as long as the mask remains, while the mask is also etched and worn out by the ion beam. This fact suggests that the depth of the pores can be increased as much as we need by increasing the thickness of the alumina layer. In reality, however, there is a limit given by the requirements of the processibility. In fact, the membrane with pores with extremely high aspect ratio is not suitable as a mask because the ion beam may not reach the bottom of the pores if the pore axes are slightly tilted from the

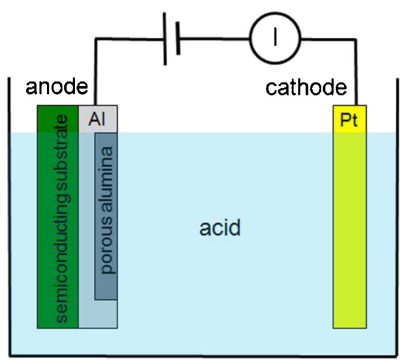

Fig. 3. An example of the conventional anodization setups.
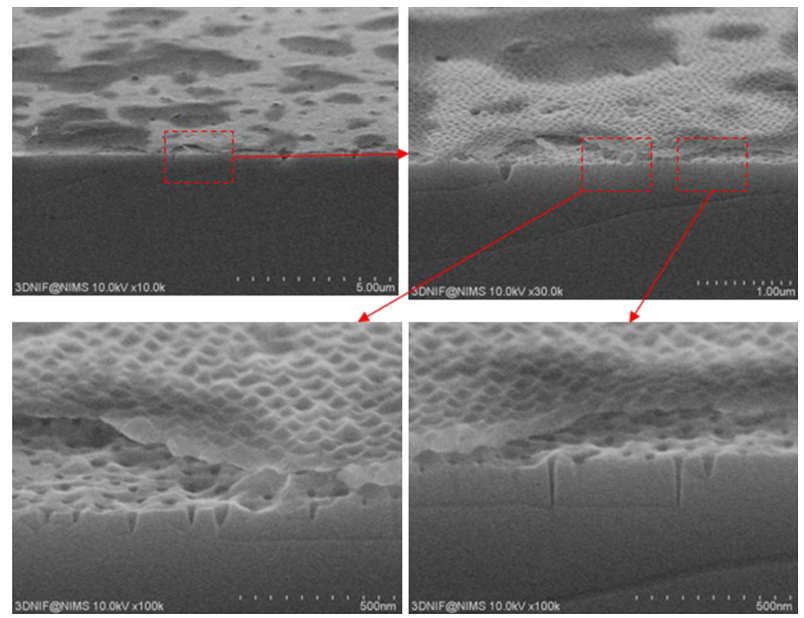

Fig. 4. SEM images of the surface of the sample after the dry etching with a nonuniform porous alumina mask prepared with a conventional setup. The upper right and lower two panels show the enlargements of the parts shown by the respective red squares.

beam direction. As a compromise between these conditions, we have empirically found that the pores can be best fabricated for the thickness of the alumina mask up to about $0.5-1.0 \mu \mathrm{m}$. Let us define the tilt angle $\theta$ as that of the pore axis from the surface normal, then the maximum $\theta$ allowed for the etching is given by $\theta_{\mathrm{m}}=\arctan \left(1 / A_{r}\right)$, where $A_{r}$ is the aspect ratio. In the present case, $A_{r}=10-20$, which yields $\theta_{\mathrm{m}}=3-6^{\circ}$. This means that the tilt angles of the pores are within about $6^{\circ}$.

\section{Current uniformization in the anodization process}

The main challenge in this method is to fabricate homogeneous nanopore arrays over the large area of the aluminum layer deposited on the semiconducting substrate. It is often the case in the conventional setup as shown in Fig. 3 that the anodization proceeds nonuniformly, which results in a partial anodization of the sample. In most cases, the anodization occurs only at the top part of the sample, but in other cases, there appear some islands (spots) of unanodized regions at the middle or the bottom of the sample. In addition, we find that the quality of the pores at the anodized part is low; Fig. 4 shows some SEM images of the upper part of the sample prepared with the conventional setup, which exhibit uneven surfaces.

These results suggest that the nonuniformity originates from that of the electric current flowing inside the aluminum layer. We find it essential that both the porous alumina and the substrate are insulators; once the top of the aluminum layer is oxidized, the current path to the rest of the aluminum layer vanishes and the anodization process stops, resulting in a nonuniform anodic alumina film. In fact, we have found in the case of a conductive 


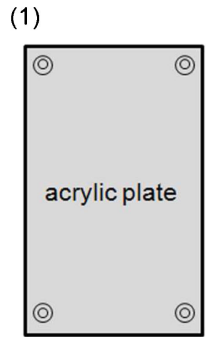

(4)

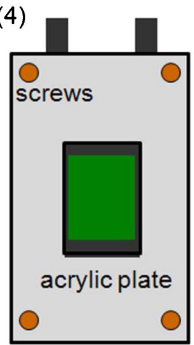

(2)

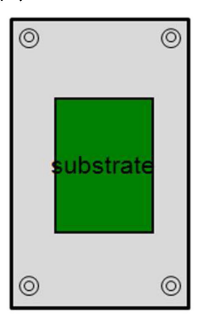

(3)

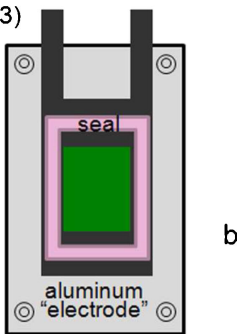

(5)

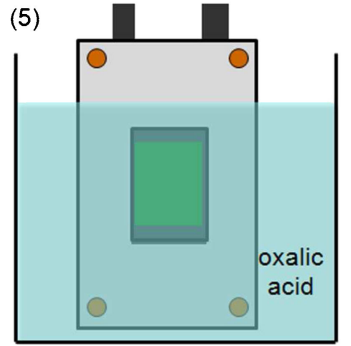

b

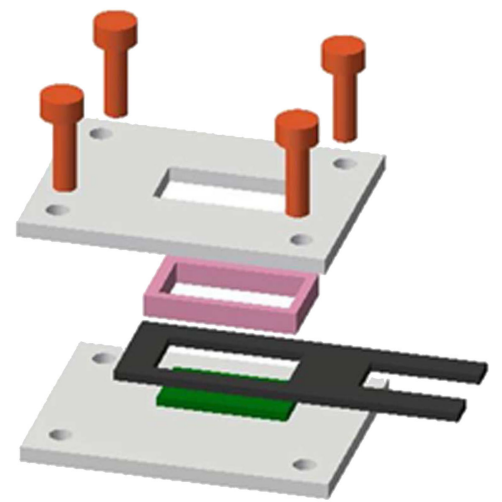

Fig. 5. (a) Schematic Illustration of the assembly of the fitting to the anode. (1) An insulating plate (e.g. acryl plate) is prepared. (2) The sample (substrate + aluminum layer) is placed on the insulating plate with the aluminum side upward. (3) A thin aluminum plate ("electrode") is placed on the sample so that the aluminum layer of the sample is enclosed by it. Then, a silicone rubber seal is set on the "electrode". (4) Another insulating plate with a square window is placed on the sample and fixed with screws. (5) The whole part is soaked into an oxalic acid, and a voltage is applied. The current is supplied to the sample uniformly through its edges, resulting in the highly homogeneous anodization. (b) A deal drawing of the fitting.
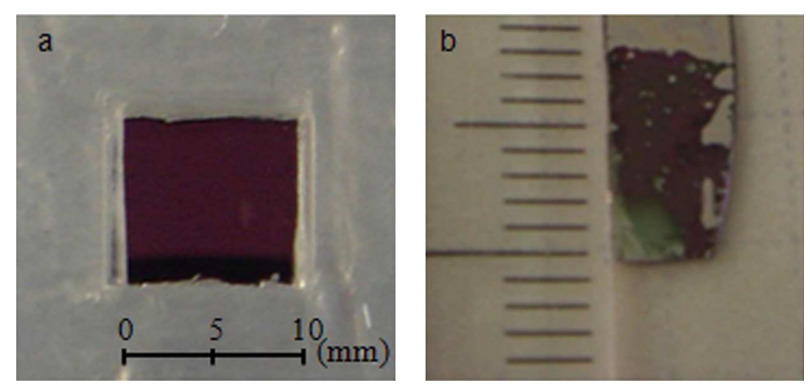

Fig. 6. (a) A uniformly anodized sample (center, dark part). The gray part around the sample is the "electrode" made of an aluminum plate, which is pressed onto the sample at the edge with a semi-transparent silicone rubber seal. (b) An example of the inhomogeneously anodized samples resulting from the insufficient contact between the "electrode" and the sample. One division of the scale next to the sample is $1 \mathrm{~mm}$.

(Si-based) substrate that the entire surface of the aluminum layer on the substrate can be homogeneously anodized even with the conventional setup.

To circumvent this problem, we have implemented a fitting to the anode, whose details are shown in Fig. 5. In this setup, the sample is enclosed with an aluminum plate, which serves as an "electrode". In addition, we have secured the contact between the "electrode" and the aluminum layer on the sample by pressing them with a silicone rubber seal. Since the current is supplied to the sample evenly from the periphery, homogeneous anodization can be realized. Figure 6(a) shows a top view of the sample described in the previous section, which suggests that a large area of the order of one square centimeter is uniformly anodized. The SEM images of the same sample (Fig. 2) demonstrate good quality of the pores. On the other hand, the process with the "electrode" insufficiently contacted to the aluminum layer on the sample has proved unsuccessful: Fig. 6(b) shows a top view of the sample, where we intentionally left the bottom part of the sample uncovered with the "electrode", which resulted in a large unanodized area at the bottom. This result demonstrates the importance of the firm contact between the "electrode" and the aluminum layer.

SEM observations suggest that in most cases the aluminum layer remains firmly contacted to the substrate even after such nonuniform anodization; therefore, a possible exfoliation of the aluminum layer from the substrate during the anodization process, even if occurs, may not be the primary origin of the nonuniform anodization. Nevertheless, since the uniform current supply to the aluminum layer is the key for the uniform anodization, we expect that the present method may be effective even in the case of the exfoliated aluminum layer.

Finally, we should note that it is crucial to anodize the "electrode" and the aluminum layer on the sample concurrently to prevent the concentration of the current. This is why we use aluminum as an "electrode" material; otherwise, the current is concentrated in either the aluminum layer on the sample or the "electrode" depending on the conductivity of the "electrode" material, which causes the nonuniform anodization.

\section{Conclusion}

We have developed a method for uniformly anodizing an aluminum layer deposited directly on a non-conductive semiconductor substrate. The implementation of the fitting to the anode causes the equalization of the current in the aluminum layer, resulting in homogeneous nanopores over the entire surface of the sample.

Acknowledgements We thank S. Nigo and S. Hashimoto for their advices. We are indebted to Materials Analysis Station of NIMS for their help in obtaining the SEM images. This work was partly supported by the Nanotechnology Network sponsored by Ministry of Education, Culture, Sports, Science and Technology (MEXT) of Japan, and a Grant-in-aid for Scientific Research by Japan Society for the Promotion of Science (JSPS). 


\section{References}

1) C. Rossi, K. Zhang, D. Estève, P. Alphonse, P. Tailhades and C. Vahlas, J. Microelectromech. Syst., 16, 919-931 (2007).

2) P. Menna, G. Di Francia and V. La Ferrara, Sol. Energy Mater. Sol. Cells., 37, 13-24 (1995).

3) V. Kochergin and H. Foll, "Porous Semiconductors: Optical Properties and Applications", Engineering Materials and Processes Series, Springer-Verlag, London (2009).

4) H. Masuda and M. Satoh, Jpn. J. Appl. Phys., 35, L126-L129 (1996).
5) H. Masuda and K. Fukuda, Science, 268, 1466-1468 (1995).

6) S. Shingubara, O. Okino, Y. Murakami, H. Sakaue and T. Takahagi, J. Vac. Sci. Technol., B, 19, 1901-1904 (2001).

7) G. Meng, T. Yanagida, K. Nagashima, T. Yanagishita, M. Kanai, K. Oka, A. Klamchuen, S. Rahong, M. Horprathum, B. Xu, F. Zhuge, Y. He, H. Masuda and T. Kawai, RSC Adv., 2, 1061810623 (2012).

8) H. Masuda, H. Yamada, M. Satoh, H. Asoh, M. Nakao and T. Tamamura, Appl. Phys. Lett., 71, 2770-2772 (1997). 